\title{
Studies of plasmonic hot-spot translation by a metal-dielectric layered superlens
}

Thoreson, Mark D.; Nielsen, Rasmus Bundgaard; West, Paul R.; Kriesch, Arian; Liu, Zhengtong; Fang, Jieran; Kildishev, Alexander V.; Peschel, Ulf; Shalaev, Vladimir M.; Boltasseva, Alexandra

\section{Published in:}

Proceedings of SPIE - The International Society for Optical Engineering

Link to article, DOI:

$10.1117 / 12.894225$

Publication date:

2011

Document Version

Publisher's PDF, also known as Version of record

Link back to DTU Orbit

Citation (APA):

Thoreson, M. D., Nielsen, R. B., West, P. R., Kriesch, A., Liu, Z., Fang, J., Kildishev, A. V., Peschel, U., Shalaev, V. M., \& Boltasseva, A. (2011). Studies of plasmonic hot-spot translation by a metal-dielectric layered superlens. Proceedings of SPIE - The International Society for Optical Engineering, 8093, 80931J. https://doi.org/10.1117/12.894225

\section{General rights}

Copyright and moral rights for the publications made accessible in the public portal are retained by the authors and/or other copyright owners and it is a condition of accessing publications that users recognise and abide by the legal requirements associated with these rights.

- Users may download and print one copy of any publication from the public portal for the purpose of private study or research.

- You may not further distribute the material or use it for any profit-making activity or commercial gain

- You may freely distribute the URL identifying the publication in the public portal 


\title{
Studies of plasmonic hot-spot translation by a metal-dielectric layered superlens
}

\author{
Mark D. Thoreson ${ }^{\mathrm{a}, \mathrm{b}}$, Rasmus B. Nielsen ${ }^{\mathrm{c}}$, Paul R. West ${ }^{\mathrm{a}}$, Arian Kriesch ${ }^{\mathrm{b}, \mathrm{d}, \mathrm{e}}$, Zhengtong Liu ${ }^{\mathrm{f}}$, Jieran \\ Fang $^{\mathrm{a}}$, Alexander V. Kildishev ${ }^{\mathrm{a}}$, Ulf Peschel ${ }^{\mathrm{d}, \mathrm{e}}$, Vladimir M. Shalaev ${ }^{\mathrm{a}}$ and Alexandra Boltasseva, ${ }^{\mathrm{a}, \mathrm{b}, \mathrm{c}}$ \\ ${ }^{a}$ Birck Nanotechnology Center and School of Electrical and Computer Engineering, Purdue \\ University, 1205 W. State St., West Lafayette, IN 47907 USA, mthoreso@purdue.edu \\ ${ }^{b}$ Erlangen Graduate School of Advanced Optical Technologies (SAOT), Universität Erlangen- \\ Nürnberg, Paul-Gordan-Str. 6, 91052 Erlangen, Germany \\ ${ }^{\mathrm{c}}$ DTU Fotonik, Department of Photonics Engineering, Technical University of Denmark, Building \\ 343, DK-2800 Kongens Lyngby, Denmark \\ ${ }^{\mathrm{d}}$ Max Planck Institute for the Science of Light, Günther-Scharowsky-Str. 1, 91058 Erlangen, \\ Germany \\ ${ }^{\mathrm{e}}$ Cluster of Excellence Engineering of Advanced Materials (EAM), Universität Erlangen-Nürnberg, \\ Nägelsbachstraße 49b, 91052 Erlangen, Germany \\ ${ }^{\mathrm{f}}$ Institute of High Performance Computing, 1 Fusionopolis Way, \#16-16 Connexis, Singapore \\ 138632
}

\begin{abstract}
We have studied the ability of a lamellar near-field superlens to transfer an enhanced electromagnetic field to the far side of the lens. In this work, we have experimentally and numerically investigated superlensing in the visible range. By using the resonant hot-spot field enhancements from optical nanoantennas as sources, we investigated the translation of these sources to the far side of a layered silver-silica superlens operating in the canalization regime. Using near-field scanning optical microscopy (NSOM), we have observed evidence of superlens-enabled enhanced-field translation at a wavelength of about $680 \mathrm{~nm}$. Specifically, we discuss our recent experimental and simulation results on the translation of hot spots using a silver-silica layered superlens design. We compare the experimental results with our numerical simulations and discuss the perspectives and limitations of our approach.
\end{abstract}

Keywords: superlens, canalization, silver, silicon dioxide, finite-difference time-domain simulations, near-field scanning optical microscopy

\section{INTRODUCTION}

In 1873, Ernst Abbe postulated that it is impossible to focus light below a certain limit due to diffraction effects. Termed the Abbe limit or the diffraction limit, this idea was fundamentally challenged in 2000 with the proposal of a subdiffraction "superlens" by Pendry [1], which was based on the 1968 paper on negative-index materials by Veselago [2]. Pendry's initial superlens would in principle work for propagating (or far-field) waves and would allow unlimited resolution in the image of an object. This superlens design required some rather strict conditions on the permittivity and permeability of the superlens material, however. Pendry's proposal also included a simplified, near-field superlens design that required only a negative permittivity, rather than simultaneously negative permittivity and permeability. Near-field superlensing was experimentally demonstrated in 2005 by Fang and coworkers [3] and nearly simultaneously by Melville and Blaikie [4], followed by others [5]. However, the practical applications of near-field superlenses are still quite limited due to the fact that the permittivities of the lens and the surrounding medium must be equal in sign and opposite in magnitude. Since many metals have negative permittivities in the visible range, they are a natural choice for superlenses. For superlensing operation, the matching condition $\varepsilon_{\text {metal }}=-\varepsilon_{\text {dielectric }}$ must be satisfied [1] for the real parts of the permittivities. For dispersive materials, this condition means that superlens operation can only be achieved at a

Metamaterials: Fundamentals and Applications IV, edited by Allan D. Boardman, Nader Engheta, Mikhail A. Noginov, Nikolay I. Zheludev, Proc. of SPIE Vol. 8093, 80931J · C 2011 SPIE · CCC code: 0277-786X/11/\$18 · doi: 
single wavelength for each material system. In the case of the silver-polymer combination used in many of the initial near-field superlens demonstrations, the matching condition is met at a wavelength of around $365 \mathrm{~nm}$ [2-4]. However, superlensing in the visible or near-infrared wavelength range promises a number of exciting applications. Several solutions have been proposed to bring superlensing to this spectral range, including the use of metal-dielectric composites (MDCs) [6-9] and multilayer lens structures [10-15]. These solutions allow the designer to select or "tune" the superlens operational wavelength by adjusting the fabrication procedure appropriately. Note that in using the term "tune" here we mean that the operational wavelength of the superlens can be selected prior to fabrication. If such a tunable near-field superlens could be achieved, it would open up many promising applications based on the spatial translation (or transfer) of highly localized, enhanced electromagnetic fields [16-23] to the other side of a superlens [24]. In this scheme, the localized and enhanced electromagnetic fields (or hot spots) could be created by optical nanoantennas [25-28], and the hot-spot transfer with a superlens could be useful in applications such as surface-enhanced Raman spectroscopy (SERS) [29] or enhanced fluorescence microscopy [30]. Optical hot spots are important for a range of important bio-, chemical- and medical-sensing applications, including surface-enhanced Raman scattering [11] and enhanced fluorescence [28]. It has also been shown theoretically that an optical hot spot can be translated through a nearfield superlens [13]. Such a setup is preferable to direct contact with a metal, which has been shown to cause structural and functional changes in biomolecules [31], including denaturation of proteins [32]. Furthermore, very close proximity with metal nanoparticles can cause fluorescence to be quenched rather than enhanced [33].

Although we have studied both MDCs and multilayer superlens designs in our research, here we focus on the latter category, specifically lamellar, multilayer metal-dielectric devices for superlensing in the visible range. Therefore, as a side note, we briefly mention some of the experimental [34] and numerical simulation [35] results on our work with MDC films. Metal-dielectric composites or MDCs are materials that consist of a random mixture of subwavelength metallic in a dielectric host (or alternatively, small dielectric particles in a metallic host); such materials are sometimes called cermets. In principle, according to effective-medium theories, MDCs permit a designer to adjust the effective permittivity of the mixture through selecting the constituent materials and the relative volume fractions. Ideally, such a MDC material should produce a selectable effective permittivity at any wavelength in a wide range while maintaining low loss. We have observed that it is indeed possible to adjust the real part of the permittivity of a superlens based on MDC films, and hence the superlens operational wavelength can be tailored for a particular application simply by altering the volume ratio between the metal and dielectric components in the composite. However, we also discovered that passive MDC designs are inherently too lossy to be useful in real superlens applications [34, 35]. It is due to this overwhelming loss that we turned our attention to lamellar, multilayer superlens designs.

Single-layer and multilayer near-field superlenses have been studied by a number of researchers in recent years. Most experimental studies have focused on the plasmonic operational regime, which is limited to the near-UV range for noble metals and common dielectrics. In this regime, the matching condition is met directly through the careful selection of the metal and dielectric materials, and the wavelength of operation is not adjustable once the material combination is determined. Numerical studies have also predicted the existence of a canalization regime in which superlensing occurs in a highly anisotropic, layered, metal-dielectric system at wavelengths away from the plasmon resonance and into the visible range. This canalization regime has been experimentally observed in the microwave range. In this work, we have experimentally and numerically investigated superlensing in the visible range. Using the resonant hot-spot enhancements from arrays of optical nanoantennas as sources, we investigated the translation of these sources to the far side of a layered silver-silica superlens operating in the canalization regime. With the technique of near-field scanning optical microscopy, we have observed evidence of superlens-enabled enhanced-field translation at a wavelength of about 680 $\mathrm{nm}$. The experimental evaluation of the fabricated samples included near-field scanning optical microscopy (NSOM), scanning electron microscopy (SEM), atomic force microscopy (AFM) and far-field spectroscopy. We have also studied the samples extensively using FDTD simulations to evaluate their near-field electromagnetic behavior. Finally, we summarize and compare our experimental results with respective numerical simulations and discuss the perspectives and limitations of our approach.

\section{METHODS}

Lamellar, multilayered metal-dielectric systems such can be used for wavelength-selectable near-field superlenses in the canalization regime. A schematic of such a multilayered lens design is shown in the left panel of Figure 1, and an SEM image of a prototype multilayer structure is shown in the right panel of the figure. These multilayered metal-dielectric systems can be considered to be uniaxial crystals that exhibit a flat, hyperbolic dispersion relation [10,11]. Multilayered 
systems have been extensively studied theoretically, and their lensing and optical properties have been discussed by a number of authors [11-15].
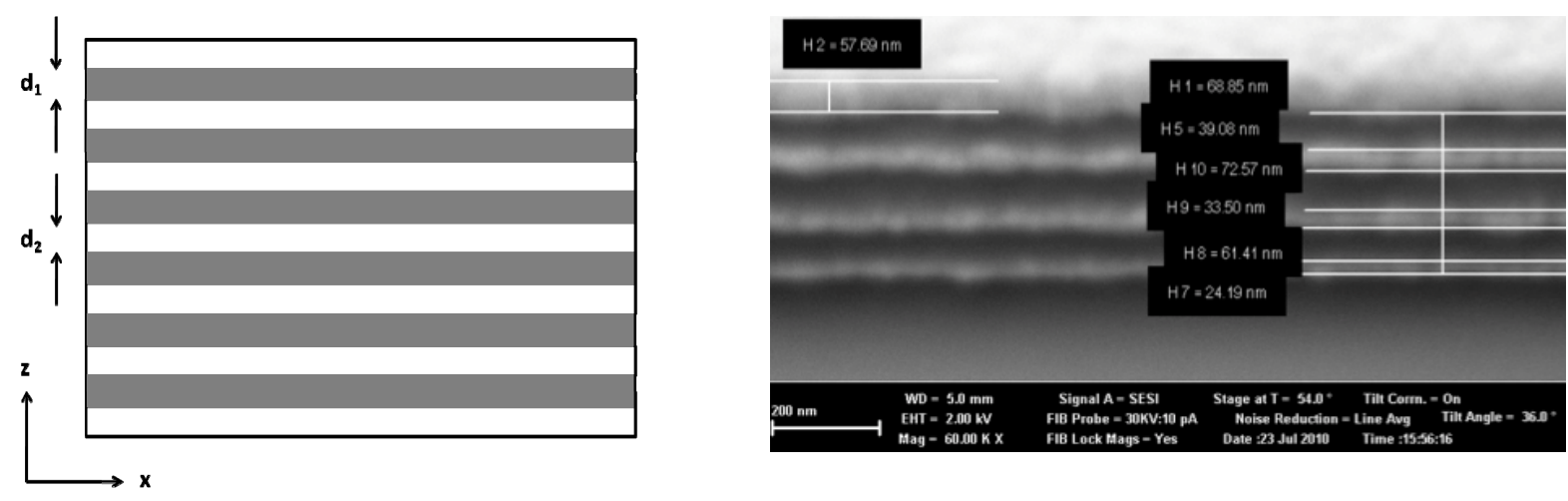

Figure 1: Schematic of a lamellar, alternating multilayer metal-dielectric system (left). The metal is white, the dielectric is gray, and the layers are subwavelength in thickness. This scheme provides a method of adjusting the operational wavelength of a superlens. A cross-section SEM view of a silver-silica prototype superlens design with layer thicknesses labeled (right).

A metal-dielectric multilayered system with subwavelength layer thicknesses exhibits an anisotropic permittivity characterized by the dispersion function [11]

$$
\varepsilon_{x}=\varepsilon_{y}=\frac{\varepsilon_{d} d_{1}+\varepsilon_{m} d_{2}}{d_{1}+d_{2}}, \quad \varepsilon_{z}=\left(\frac{\varepsilon_{d}^{-1} d_{1}+\varepsilon_{m}^{-1} d_{2}}{d_{1}+d_{2}}\right)^{-1},
$$

where $\varepsilon_{\mathrm{d}}$ and $\varepsilon_{\mathrm{m}}$ are the bulk permittivities of the dielectric and metal constituents, respectively, and the thicknesses are as labeled in Figure 1. In superlensing applications, we are interested in obtaining a system with a parallel permittivity of unity for impedance matching $\left(\varepsilon_{\mathrm{x}}=\varepsilon_{\mathrm{y}}=1\right)$ and an infinite perpendicular permittivity $\left(\varepsilon_{\mathrm{z}} \rightarrow \infty\right)$. This is known as the canalization regime [11]. In practice, however, due to losses and material limitations, both conditions cannot be fulfilled simultaneously. These multilayer films are not restricted to operation at a single wavelength for a given material combination; the operational wavelength can be tuned simply by altering the relative thicknesses of the metal and dielectric layers.

\section{Nanoantenna pattern design and fabrication}

As stated above, we were primarily interested in studying the ability of a near-field superlens to transfer field-enhanced optical hot spots, and hence our first step was to design an array of optical nanoantennas to produce these hot-spot sources. The antenna design was based on pairs of identical rhombus-shaped metal structures with a narrow gap between the two. It is in this narrow gap where electromagnetic energy is localized and where the hot spot is produced. These very high local field intensities are created between antenna pairs only when the pair is illuminated with correctly polarized light (that is, light that is polarized parallel to the long axis of the antenna pair). This antenna design choice was selected based on existing expertise from previous work [28].

Schematic designs of our antenna array are shown in Figure 2. Each individual antenna has a characteristic size X, which is the length of the minor axis. In our case, $X$ was about $80 \mathrm{~nm}$. The major axis is twice as long $(160 \mathrm{~nm})$, and the second antenna is placed along this axis, with a gap of $20 \mathrm{~nm}$ separating the two. The antenna pairs are spaced with a periodicity of $4 \mathrm{X}(320 \mathrm{~nm})$ along the minor axis and $8 \mathrm{X}(640 \mathrm{~nm})$ along the major axis (see Figure 2). 
Top view
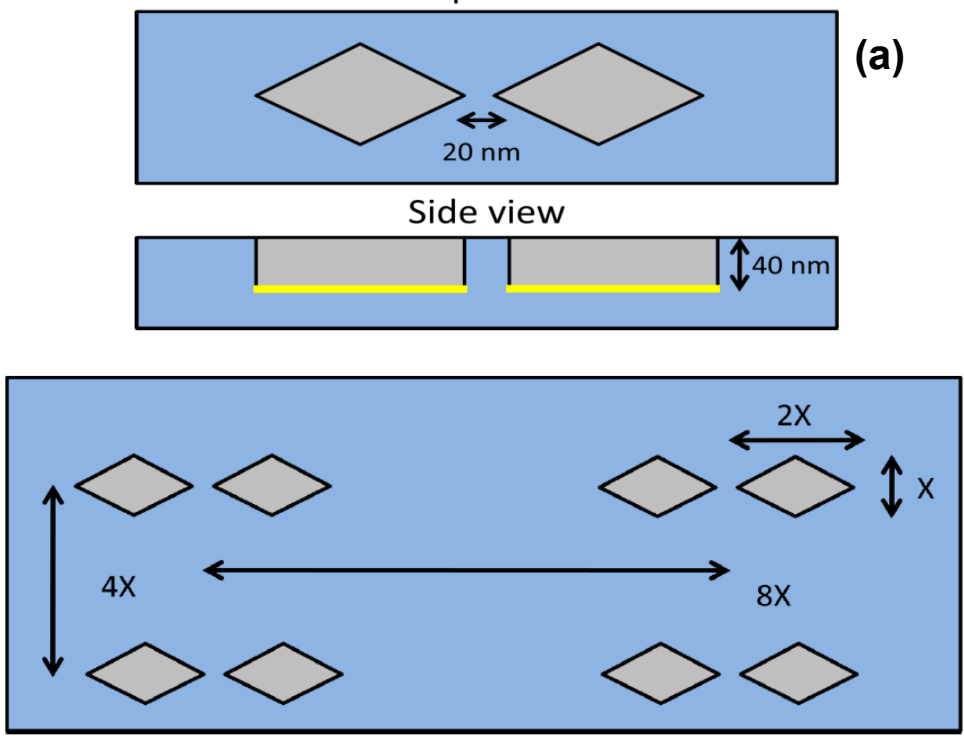

(b)

Figure 2: Schematics of the basic nanoantenna design. (a) A nanoantenna pair in top view and cross section. Two 40-nm thick rhombus-shaped antennas form a single pair and are spaced $20 \mathrm{~nm}$ apart. The antenna pair is embedded into a quartz substrate. (b) The individual antenna pairs are part of larger arrays. The size of the individual antennas is related to the spacing of the array. The size parameter X can be varied in order to achieve different resonance wavelengths. In the arrays we studied in this work, $\mathrm{X}$ was $80 \mathrm{~nm}$.

The nanoantenna fabrication was performed by electron-beam lithography (EBL) on a quartz substrate in large, $500 \times$ $500 \mu \mathrm{m}^{2}$ arrays in order to permit far-field optical characterization the final samples. Following EBL photoresist patterning and development, a reactive-ion etch procedure was used to create 40-nm holes in the quartz substrate. Next, a 1-nm layer of Ge followed by a 40-nm layer of Ag was deposited into these holes, resulting in a flatter topography than would be possible without etching. For simplicity, light polarized along the major axis (across the gap) will be referred to as parallel or x-polarized, while light polarized along the minor axis will be referred to as perpendicular or y-polarized light. The antenna array was designed to be resonant under parallel-polarized light at a wavelength of about $633 \mathrm{~nm}$.

\section{Superlens design}

The superlens design was based on finite-element model (FEM, COMSOL Multiphysics) calculations and modeling using a chromium grating to simulate lens performance at a resolution of $100 \mathrm{~nm}$, which was the grating half-pitch. The results from these initial numerical simulations indicated that subwavelength resolution might be possible without impedance matching, with $\lambda / 6$ resolution achieved for a single-layer silver lens with a $\mathrm{SiO}_{2}$ host. The highest contrast was achieved for a three-layer superlens design. However, the intensity transmission through the sample was expected to be too low for NSOM measurements for this configuration. As a result, a single-layer design was chosen since it provided the best compromise between contrast and transmission. The superlens design consisted of a 20 -nm silver layer covered on both sides by $21-\mathrm{nm} \mathrm{SiO}_{2}$ layers. While not included in the model, a 1-nm germanium adhesion layer was added below the silver layer in order to lower the silver roughness and decrease the loss. The germanium layer itself is not expected to add much loss due to the very low thickness and relatively low loss at $633 \mathrm{~nm}$ [36].

We designed and fabricated three different types of samples for our experimental investigations. Although the three sets of samples have exactly the same nanoantenna design, the regions above samples differ in their overlayer designs (see Figure 3). The first sample type was simply uncovered or "bare" nanoantennas with no overlayer. In the superlens samples, the nanoantennas are covered with $21 \mathrm{~nm}$ of silica, $20 \mathrm{~nm}$ of silver, and another $21 \mathrm{~nm}$ of silica. In the final type of sample, the nanoantennas were covered with a $62-\mathrm{nm}$ silica layer. This is an equivalent thickness to that in the superlens samples, and hence these samples were used as reference samples. The reference and bare nanoantenna samples were used to compare the near-field intensity distributions to those of the superlens samples. In all samples, the 
overlayers were fabricated using electron-beam evaporation. The cross-sections of the three sample types are shown schematically in Figure 3.
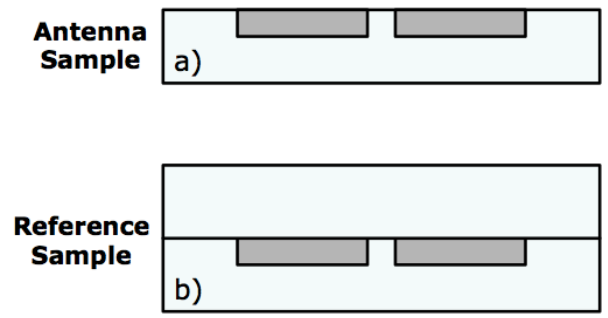

$62 \mathrm{~nm} \mathrm{SiO}{ }_{2}$ 40nm Ag Antennas Quartz substrate

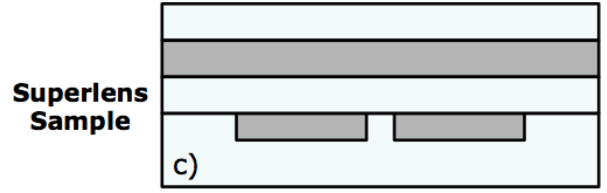

$21 \mathrm{~nm} \mathrm{SiO}{ }_{2}$ $20 \mathrm{~nm}$ Ag Superlens $21 \mathrm{~nm} \mathrm{SiO}$ 40nm Ag Antennas Quartz substrate

Figure 3: Cross-section of the three types of samples examined in this study. a) the "bare antenna" sample, b) the "reference" sample with a dielectric spacer above the antennas, and c) the "superlens" sample with layered metal and dielectric films above the antenna arrays.

\section{SAMPLE CHARACTERIZATION}

\section{Structural characterization}

We performed a number of tests in order to evaluate the quality of the fabricated samples and to characterize their properties. These tests were focused on the antenna shape as well as the planarization and quality of the silver layer. We first used scanning electron microscope (SEM) imaging to study the uncoated antenna arrays, an example of which can be seen in Figure 4. The antenna shape was generally well reproduced, though the finite size of metallic grains does lead to a rounding of the edges. As we will show, this rounding was taken into account in our numerical simulations. In general, however, the nanoantenna elements were all generally very uniform with no missing antenna pairs.

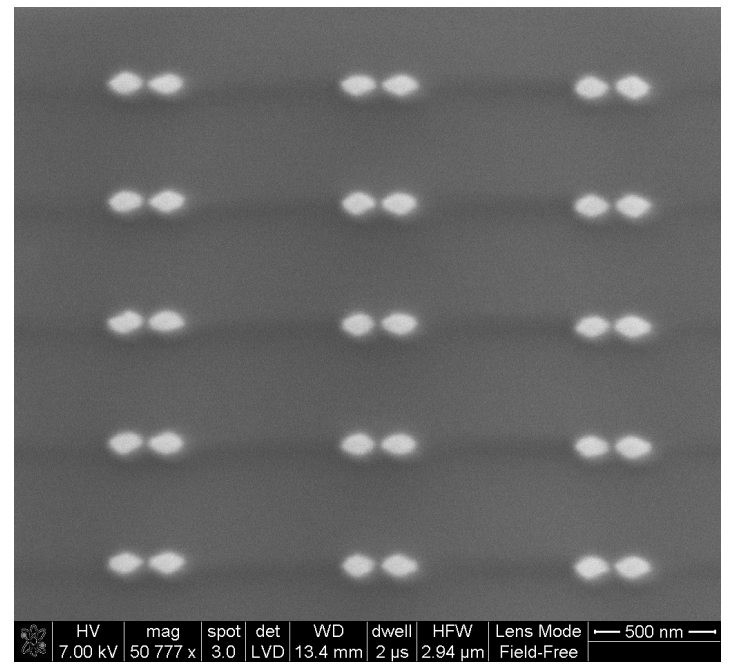

Figure 4: SEM image of a nanoantenna array $(X=80 \mathrm{~nm})$. The pattern is well reproduced with a gap size around $20 \mathrm{~nm}$. Some minor variations in antenna shape can be observed, which can be ascribed to the finite metal grain size. The slight rounding of the corners of the nanoantennas is accounted for in our numerical simulations. 
We also used atomic force microscope (AFM) imaging to investigate the roughness and surface quality of the embedded nanoantennas. Our goal was to produce a flat final surface with minimized height deviations between the antennas and the surrounding substrate within the antenna arrays. The AFM results (not shown here) indicate that the embedding was generally very good, and the average height variation between the antennas and the substrate was less than $5 \mathrm{~nm}$ RMS. In addition, we studied the quality of the deposited 35-nm silver / 1-nm germanium superlens layers with AFM. This was done on a test sample with no $\mathrm{SiO}_{2}$ cover layer in order to allow direct access to the silver material. The results show good film uniformity with a small grain size and a low roughness of $0.5 \mathrm{~nm}$ RMS. For comparison purposes, we also deposited 35-nm silver films directly on $\mathrm{SiO}_{2}$ with no germanium adhesion layer. In this case, the AFM results show a much higher surface roughness of $2.7 \mathrm{~nm}$ RMS.

\section{Optical Characterization}

The nanoantenna array was characterized optically via far-field transmission measurements using a supercontinuum light source (Koheras) with a broad emission range from $500 \mathrm{~nm}$ to $2000 \mathrm{~nm}$. The light was focused on the sample with a focus size of 200-300 $\mu \mathrm{m}$, and a linear polarizer was used to adjust the incident light polarization. After passing through the sample, the light was refocused and coupled into a spectrometer. Background reference measurements were taken through the sample in an area free of visual defects and outside of the nanoantenna array. The reference intensities were then subtracted from the array intensities in order to give a relative transmission measurement.

As expected from theory [26, 37], a strong resonance was seen for parallel-polarized (x-polarized) light, and it is this resonance that is of interest in our work. For the bare antenna array with a size parameter of $X=80 \mathrm{~nm}$, the resonance occurred at a wavelength of $620 \mathrm{~nm}$, which is close to the expected $633-\mathrm{nm}$ resonant wavelength. A weaker resonance was seen for light polarized orthogonal to the long axis of the antennas pairs (perpendicular or y polarization). This weaker resonance wavelength was blue-shifted compared to that of the parallel polarization. Optical transmission measurements were also performed after depositing the reference silica layer or the silver/silica superlens layers on top of the bare antennas, and we observed significant changes in the transmission spectra. For the reference sample, a red shift of about $40 \mathrm{~nm}$ was observed in the resonance position. This shift was expected, since the $\mathrm{SiO}_{2}$ covering increases the refractive index around the antennas as compared to air. For the superlens-covered sample, however, the red shift was slightly larger $(45 \mathrm{~nm})$. In fact, when we collected spectra from nanoantenna arrays with different size parameters, we found that the resonant wavelength shift for silica-coated antennas was about $40 \mathrm{~nm}$ for all arrays, while the shift for superlens-covered arrays depended on the initial resonance position. Antenna arrays with an initial resonance of about $500 \mathrm{~nm}$ showed no resonance shift with the addition of the superlens, while antennas with a resonance around $700 \mathrm{~nm}$ shifted by as much as $100 \mathrm{~nm}$ when we added the superlens layers. We attribute this to coupling between the antennas and silver superlens layer. As a result of this shift, the reference samples have a different resonance wavelength than the superlens samples, even for otherwise identical antennas. Around our wavelength of interest $(\sim 633 \mathrm{~nm})$, the difference is slight, but for antennas outside this range the difference can be significant, which complicates any direct comparisons between the superlens and reference samples.

\section{Near-field optical characterization}

We performed a large number of NSOM scans in order to study the near-field intensity distribution on the samples. These measurements were carried out at The Max Planck Institute for the Science of Light and Friedrich-AlexanderUniversität (Erlangen, Germany) and at Purdue University (West Lafayette, Indiana, USA). The NSOM setup in Erlangen was equipped with an acousto-optic tunable filter in combination with a supercontinuum light source, which enabled the selection of any incident-light wavelength from $450 \mathrm{~nm}$ to $700 \mathrm{~nm}$. The setup also included a linear polarizer, and NSOM measurements were taken with a highly modified Nanonics 4000 system. The NSOM setup at Purdue was a Nanonics 2000 system but was limited to 633-nm light and did not have polarization control in our measurements. In both systems we used metal-coated aperture NSOM tips with sizes ranging from $150 \mathrm{~nm}$ down to 50 $\mathrm{nm}$, and in both systems we also used avalanche photodiodes (APDs) for detecting the collected light intensity. Several types of NSOM scans are possible, with each type providing different near-field information about the sample. Schematics of three main NSOM modes are shown Figure 5. In reflection-mode NSOM (Figure 5, left panel), the sample is illuminated in the near field via the tapered-fiber NSOM tip, and the far-field reflected or scattered light is measured from the sample. In transmission-mode NSOM (Figure 5, middle panel), the sample is again illuminated in the near field with the tapered-fiber tip, but in this case the transmitted light is collected in the far-field. In our measurements we used 
collection mode NSOM scanning (Figure 5, right panel). In this case, the sample is illuminated from the bottom with farfield light that is transmitted through the sample, and the near-field intensity is collected with the NSOM tip.

Reflection Mode

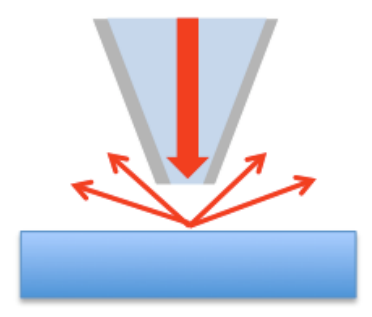

Transmission Mode
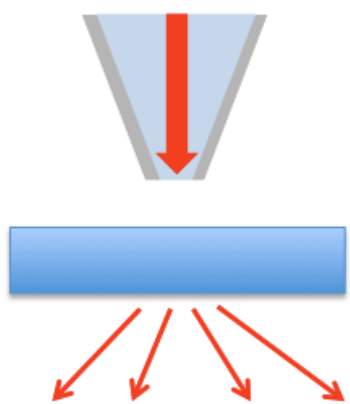

Collection Mode
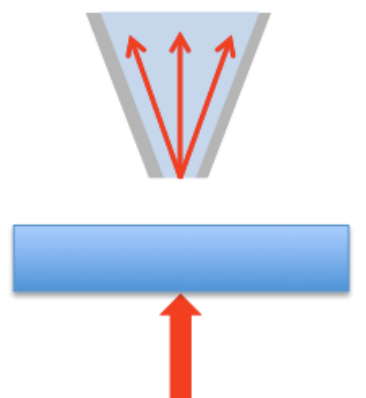

Figure 5: Three types of NSOM scanning modes. The thick arrow represents illumination of the sample. The thin arrows represent the collected or measured light. In this work we focused on collection mode NSOM (right panel) for our near-field measurements.

With the NSOM system in Erlangen, we performed numerous NSOM measurements on the nanoantenna array with a size parameter $X=80 \mathrm{~nm}$, and scans were taken at wavelengths ranging from $530 \mathrm{~nm}$ to $680 \mathrm{~nm}$ in steps of $10 \mathrm{~nm}$ and with both parallel and perpendicular light polarizations. At Purdue, measurements were done at $633 \mathrm{~nm}$ with unpolarized light. In both systems, the measurements were done on superlens samples as well as on reference samples and bare nanoantenna samples. The scan sizes were either $5 \times 5$ or $10 \times 10 \mu \mathrm{m}^{2}$. The resolution was kept constant at 128 data points per $5 \mu \mathrm{m}$.

We note that we have observed some complications in interpreting our experimental NSOM results. These issues are related to several factors, such as the fact that the fiber tips experience gradual degradation over time. In addition, there are necessary manual alignments in both of our NSOM systems, which is another source of discrepancies in comparing the data from one sample to the next. When we combine these concerns with the automatic intensity rescaling performed by the Nanonics software, we conclude that the measured intensity values cannot be compared directly between scans.

\section{PRELIMINARY NSOM RESULTS}

\section{Bare Antennas}

When scanning the bare antenna samples under perpendicularly polarized (y-polarized) light at $620 \mathrm{~nm}$, no resonance was observed, which is consistent with our far-field measurements. In this polarization, the antennas simply block part of the incoming light. Thus the antennas appear as regions with lower intensities, an example of which is shown in Figure 6. The resolution in the scan in Figure 6 is sufficient to see individual antenna pairs, but we cannot resolve individual antenna elements. 


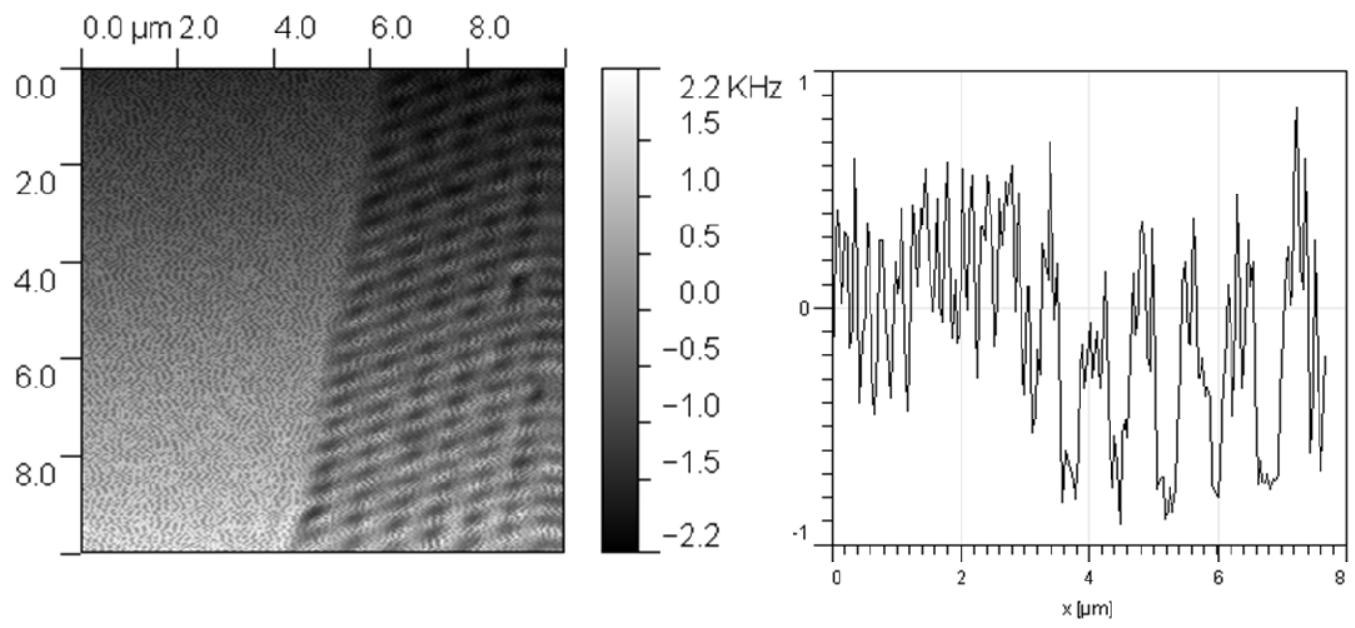

Figure 6: NSOM scan (left) and intensity cross-section (right) of a bare antenna array illuminated with perpendicularpolarized light at $620 \mathrm{~nm}$. The antenna pairs can be observed as dark regions because they block some of the light transmitted through the substrate.

When illuminating the array with light at the parallel polarization (x-polarized), a periodic enhancement of the intensity is observed. This resonance has a periodicity equal to the array periodicity, it overlaps with the light that is simply transmitted through the sample, and its peak intensity is well above that of the incident light. However, this is not the nanoantenna hot-spot resonance, which can be seen in two ways. First, when we compared the AFM and NSOM data we observed that the highest intensity regions are in the areas between the antennas, whereas the hot-spot resonance should occur in the gap in the middle of the antenna pair. Second, when looking at the edge of an array, we see that the periodic enhancement extends well beyond the boundaries of the antenna array, as seen in Figure 7. This behavior shows that the resonance is actually a propagating mode, and thus it is not the highly localized hot-spot nanoantenna resonance. Note in Figure 7 and in many of our NSOM scans that we were not able to reliably resolve the antenna pairs in the vertical direction of the image (the y-direction) because the periodicity is smaller in that direction.

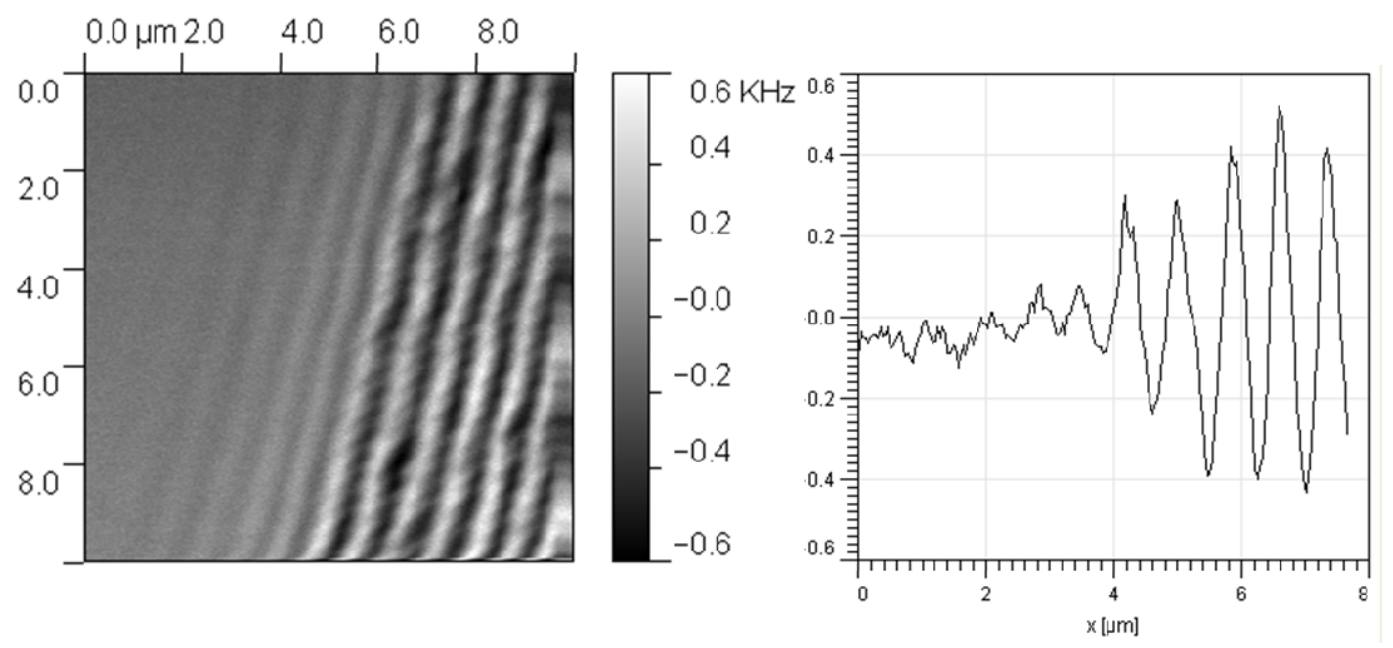

Figure 7: NSOM scan (left) and cross-section (right) of a bare nanoantenna array illuminated with parallel-polarized light at $620 \mathrm{~nm}$. In the cross-section scan, the antenna array boundary is at $4 \mu \mathrm{m}$. A periodic resonance is observed that extends well beyond the boundaries of the array and decays with distance from the array. In this resonant mode, the peak intensities are significantly higher than that of the incident light.

At this point it is important that we note some peculiarities of our antenna array pattern with respect to the expected resonance wavelength of the reference and superlens samples. It has been shown that coupled nanoantenna resonances 
die out when the distance between the antenna elements exceeds 2.5 times that of the particle size [37], which is why the nanoantenna arrays used here have a spacing of three times the particle size (corresponding to center-to-center distance of four times the size parameter $\mathrm{X}$, or about $320 \mathrm{~nm}$ for our $\mathrm{X}=80 \mathrm{~nm}$ array). However, for our silver nanoantennas this spacing had the unintended consequence of producing a periodicity $(8 \times 80=640 \mathrm{~nm})$ that is very close to the shifted resonance wavelength of the reference and superlens samples. As a result, the nanoantenna array acts essentially as a grating with a periodicity of $\lambda$ in one direction and a periodicity of $\lambda / 2$ in the other direction; such gratings have been shown previously to couple light from free space into propagating surface or waveguide modes [38,39]. We expect this grating-coupled propagating mode to travel inside the silica of the substrate itself, which could act as a waveguide due to its high refractive index compared to the surrounding air. We will discuss this grating-coupled mode in more detail in later sections.

\section{Superlens and Reference Samples}

Returning to our preliminary NSOM data, we observed a different field-enhancement distribution near the expected resonance wavelength of $665 \mathrm{~nm}(620 \mathrm{~nm}+\mathrm{a} 45-\mathrm{nm}$ shift $)$ on the superlens samples. Here a second resonance appears with the same spacing as the periodic mode described earlier but shifted by half a period. As shown in Figure 8 , this resonance is not present at $620 \mathrm{~nm}$, but it can be seen as very low intensity peaks at $650 \mathrm{~nm}$, and it is even stronger still at $680 \mathrm{~nm}$. At $680 \mathrm{~nm}$ this extra resonance mode reaches roughly the same intensity as the propagating mode, which gives it the appearance of a simple doubling in frequency. No scans were performed at longer wavelengths, since the wavelength limit of the setup had been reached, so it is not known if the intensity grows even more at longer wavelengths. 

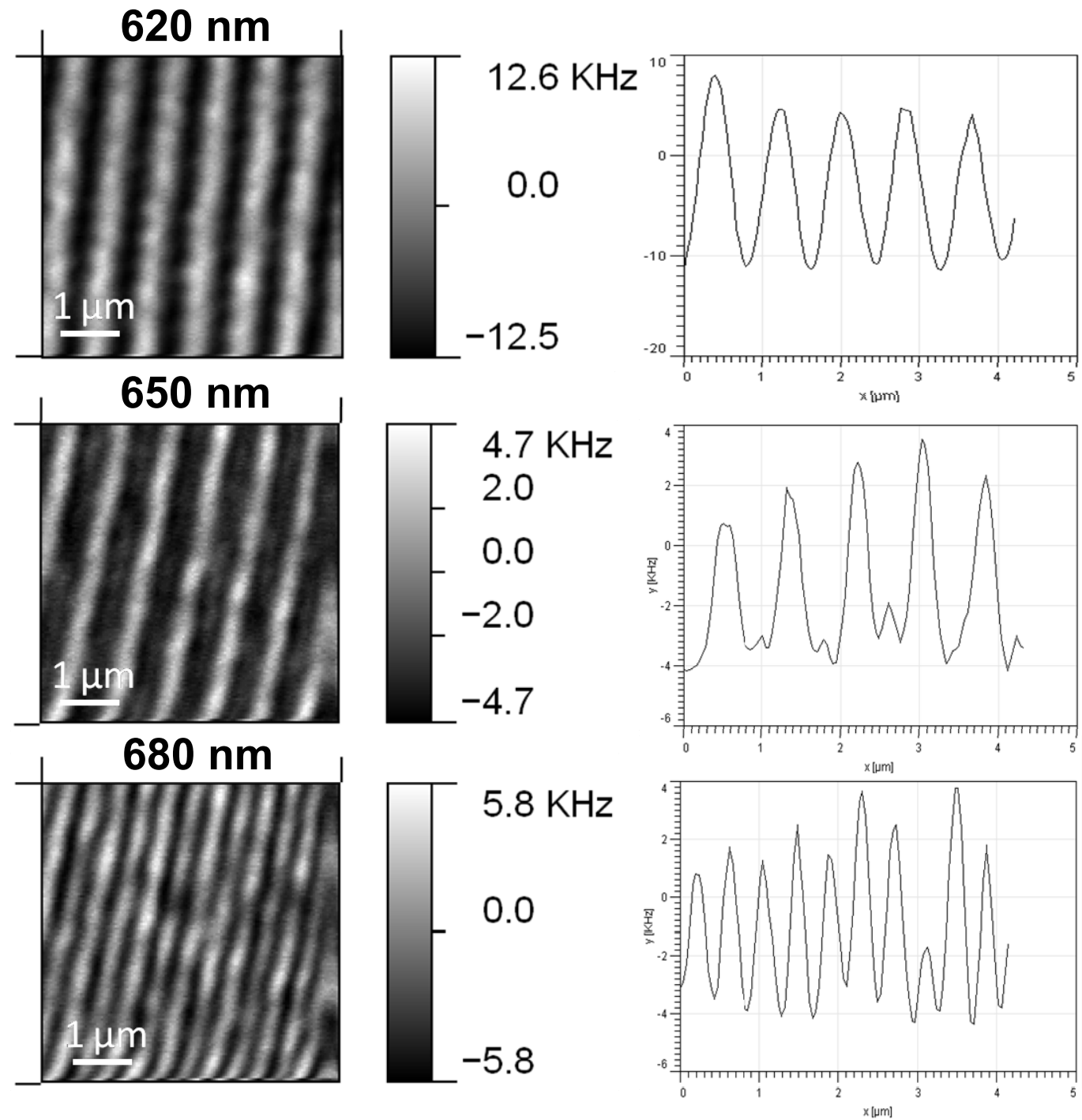

Figure 8: NSOM scans of the intensity distribution (left) and intensity cross-section (right) measured on a superlens sample for three different wavelengths. At $620 \mathrm{~nm}$ (top) only the propagating mode is seen, at $650 \mathrm{~nm}$ (middle) a weaker second mode starts to appear, and at $680 \mathrm{~nm}$ (bottom) the weaker mode has grown in strength to equal the propagating mode.

In addition to the appearance of this second resonance, we also see that the widths of the intensity peaks were reduced significantly at $680 \mathrm{~nm}$. In order to investigate the origin of this second resonance, an NSOM scan was performed using the same wavelength $(680 \mathrm{~nm})$ but with perpendicularly polarized light, and the results show no sign of the additional resonance. Furthermore, identical scans were taken on a reference sample to see if this effect was related to the silver superlens layer, and once again no secondary resonance was observed. These scans are shown in Figure 9. 


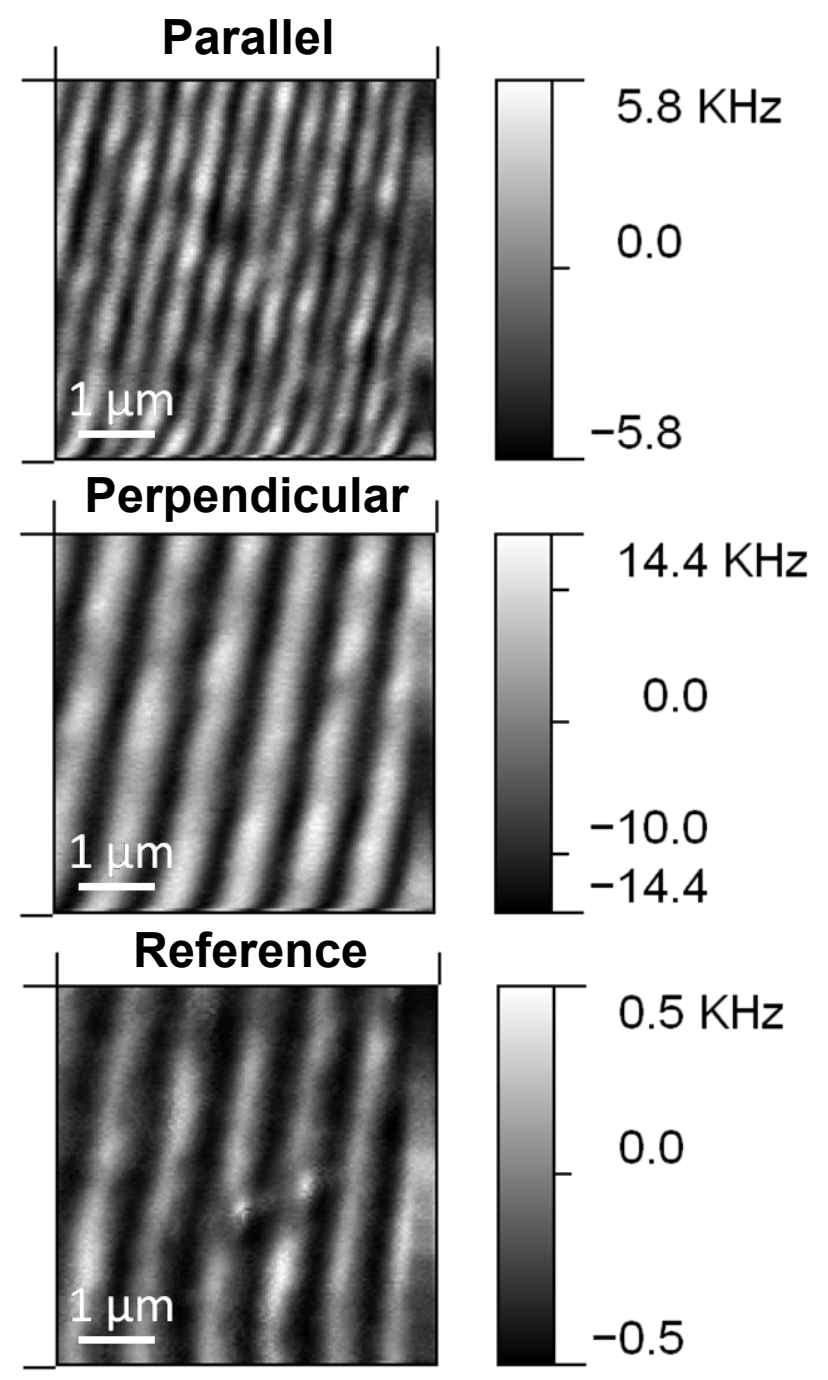

Figure 9: Comparison of the NSOM intensity distributions for the same antenna array under three different conditions. In addition to the propagating mode, a second resonance mode was observed for the parallel polarization on a superlens sample

(top panel), which caused the appearance of frequency doubling. No such mode was observed for the perpendicular polarization on the same sample (middle), or for the parallel polarization on a reference sample (bottom). All data was taken at an incident wavelength of $680 \mathrm{~nm}$.

With this preliminary NSOM data in mind, we concluded that there were two main phenomena occurring in our results. The first and most interesting was the appearance of the second resonance mode, which occurred only near a specific wavelength and in a particular polarization. The second phenomenon was the narrowing of the intensity peaks. We see three possible contributions to the near-field intensity patterns in our NSOM scans: (1) light transmitted through the sample in the bare areas between the antenna pairs, (2) the grating-coupled propagating mode, and (3) the localized field enhancement from the nanoantennas. Since the secondary resonance appears very close to the expected resonance frequency of the nanoantennas, and only at the correct polarization, it appears to be our sought-after nanoantenna hotspot resonance. Furthermore, the narrowing of the intensity peaks would be consistent with an increased extinction crosssection for the nanoantennas at resonance, which would have the effect of "stealing" light from the surrounding area. This line of reasoning suggests that this additional field enhancement is indeed the localized nanoantenna resonance, and that the superlens allows it to be resolved on the far side of the lens, which is not possible on the reference sample. However, due to the similarity of this resonance to the propagating mode, more information and additional measurements are needed to confirm this hypothesis. In the next section we discuss these further measurements as well as the numerical simulations we performed in support of our experimental results. 


\section{FDTD SIMULATIONS FOR FAR-FIELD SPECTRA AND NEAR-FIELD INTENSITY MAPS OF THE SUPERLENS AND REFERENCE SAMPLES}

\section{Near-Field Intensity Simulations and Modeling}

After obtaining our preliminary NSOM results, it was clear that numerical simulations on the antenna structures and cover layers would give us valuable insight in understanding the intensity profiles in our experimental results. We chose to use the finite-difference time-domain (FDTD) method for our simulations because the results with our new generalized dispersion model [40] are quite accurate and, with the extensive cluster computing facilities available at Purdue, full 3D simulations were possible.

As noted previously, the fabricated nanoantennas were slightly more elliptical than the initial rhombus-shaped design. Because the shape of the antenna plays a strong role in determining the overall plasmonic response, more realistic elliptical-shaped antennas were used in our simulations. The gap between antennas was fixed at $20 \mathrm{~nm}$, and the geometry of the simulated unit cell of the antenna array can be seen Figure 10.

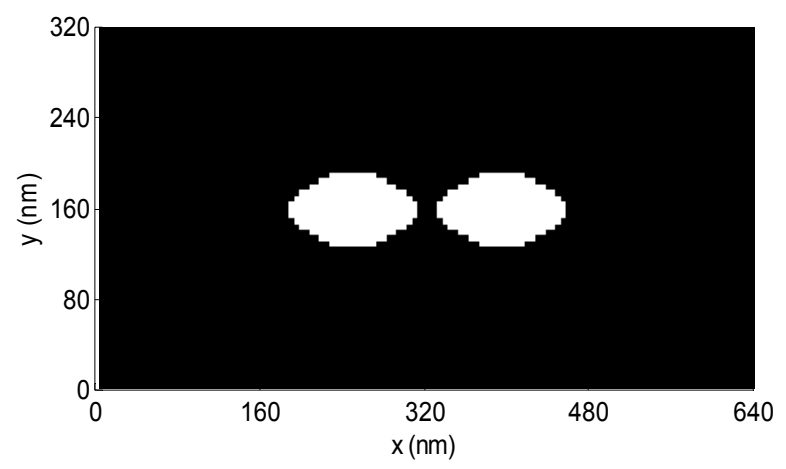

Figure 10: Elliptically-shaped nanoantenna pair used as the unit cell of the array in the FDTD simulations. The shape of these antennas corresponds more closely with the actual shape of the nanoantennas, as indicated in our SEM results. In this image, white is metal (silver) and black is dielectric material (glass).

We calculated full-wave responses of the three types of samples at a variety of incident wavelengths and polarizations. Some representative near-field intensity maps are shown below. In the simulated intensity maps, many unit cells are shown in an array in order to mimic the scan area of the experimental NSOM results. The intensities were calculated at a distance of $10 \mathrm{~nm}$ above the top surface of the sample; this was done in order to mimic the NSOM tip scanning distance. Finally, the calculated intensities were averaged with a $150-\mathrm{nm}$ circular averaging function in order to mimic the aperture area of the NSOM tip. For convenience, the ellipses in the lower left area of each image show the locations of the nanoantennas inside the simulated domain. The details of the material models used in our FDTD simulations can be found in our previous publication [35].

The nanoantenna structures were simulated for the three sample types (bare antenna sample, superlens sample, and reference sample) using fully 3D FDTD calculations. The bare antenna sample was simulated under 620-nm and 633-nm illumination schemes, corresponding to the resonance wavelength and the Purdue NSOM laser source, respectively. The superlens and reference samples were each simulated at $660 \mathrm{~nm}$ and $680 \mathrm{~nm}$, corresponding to the measured far-field resonance and the wavelength of the observed double-periodicity pattern, respectively. For each wavelength, the field distributions were calculated under parallel polarization (x-polarized, resonant case) as well as under perpendicular polarization (y-polarized, off-resonant case). For each polarization, the field intensity components were calculated along each axis direction $\left(\left|E_{x}\right|^{2},\left|E_{y}\right|^{2},\left|E_{z}\right|^{2}\right)$. The computational domain used for these simulations was $640 \mathrm{~nm}$ and $320 \mathrm{~nm}$ in the $\mathrm{x}$ and $\mathrm{y}$ directions (the $\mathrm{z}$ direction is normal to the substrate surface), and was $4000 \mathrm{~nm}$ in the $\mathrm{z}$ direction. The spatial discretization was $5 \mathrm{~nm}$, and the time discretization was about $4.814583 \times 10^{-18} \mathrm{~s}$. We used perfectly-matched layer (PML) boundaries in the $\mathrm{z}$ direction, and periodic boundary conditions (PBCs) were applied to all other sides (that is, in the $\mathrm{x}$ 
and y directions) of the simulation domain. The electric field (E-field) and magnetic field (H-field) for each unit cell were discretized, and thus the intensity of each cell was defined as $\left|\mathrm{E}_{\text {total }}\right|^{2}=\left|\mathrm{E}_{\mathrm{x}}\right|^{2}+\left|\mathrm{E}_{\mathrm{y}}\right|^{2}+\left|\mathrm{E}_{\mathrm{z}}\right|^{2}$. This total field intensity $\left(\left|\mathrm{E}_{\text {Total }}\right|^{2}\right)$ corresponds with the intensity measured experimentally with NSOM. In all our FDTD simulations, the Ge layer was omitted and the 21-nm and 62-nm thicknesses were rounded-off to $20 \mathrm{~nm}$ and $60 \mathrm{~nm}$, respectively. This was done in order to accommodate the finite-sized grid of the simulation domain. The incident wave illuminated the sample from the bottom in order to simulate the experimental situation in collection-mode NSOM measurements.

\section{Representative FDTD Near-Field Intensity Maps for Bare Antenna Sample}

In Figure 11 we show representative FDTD near-field intensity maps resulting from our numerical simulations. These intensity maps were calculated for the bare antenna sample at an incident wavelength of $620 \mathrm{~nm}$ for both polarizations. Since the intensities are not normalized among the maps, corresponding scale bars are included for each image. These maps indicate that in the parallel polarization the near-field intensity is dominated by the $\mathrm{x}$ and $\mathrm{z}$ components of the field, while in the perpendicular polarization it is the y component that dominates the total intensity. This trend is consistent for all of our calculated intensity maps at all wavelengths. Notably, the strong z-component contribution indicates a strong grating-coupled diffraction mode within the intensity distribution. This corresponds to our earlier NSOM analysis that there is a strong propagating mode in the antenna array under parallel-polarized light. 
Parallel (x-polarized) incident light at $620 \mathrm{~nm}$ Field intensity $\left|\mathrm{E}_{\mathrm{x}}\right|^{2}$
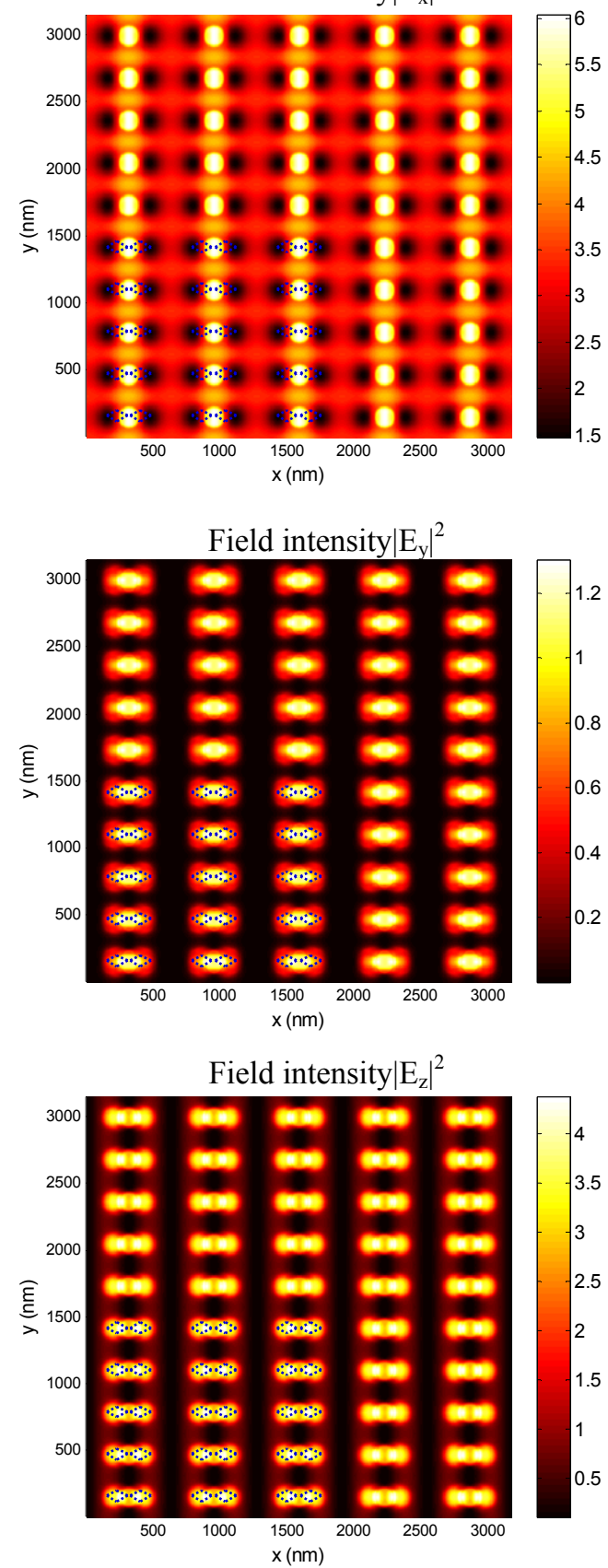

Perpendicular (y-polarized) incident light at $620 \mathrm{~nm}$

Field intensity $\left|\mathrm{E}_{\mathrm{x}}\right|^{2}$
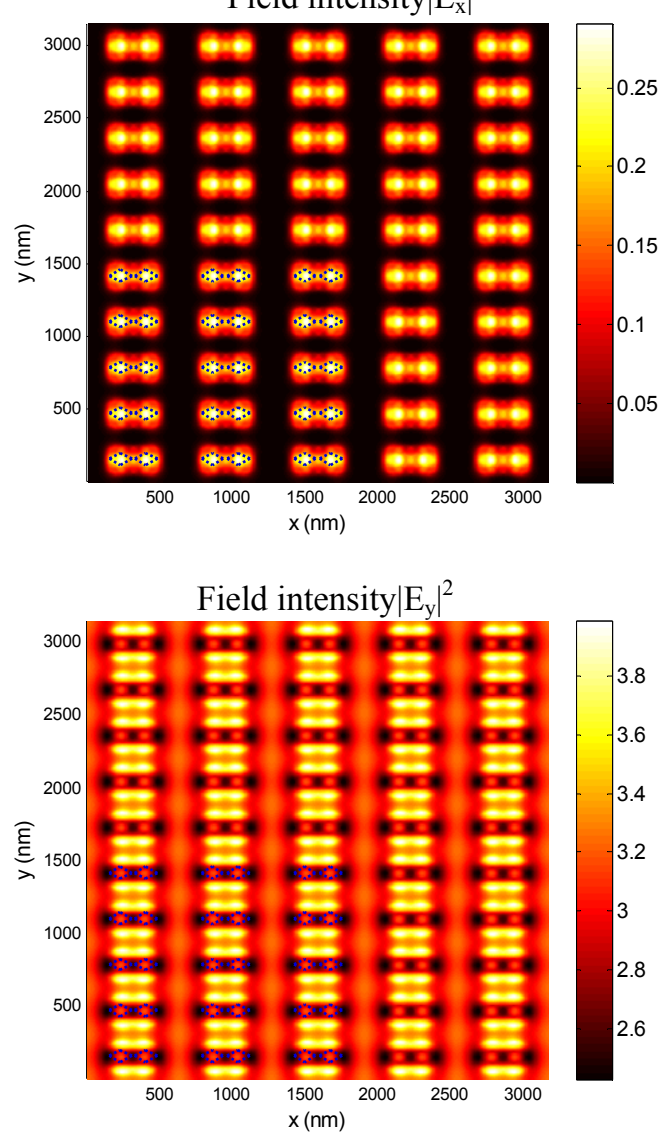

Field intensity $\left|\mathrm{E}_{\mathrm{z}}\right|^{2}$

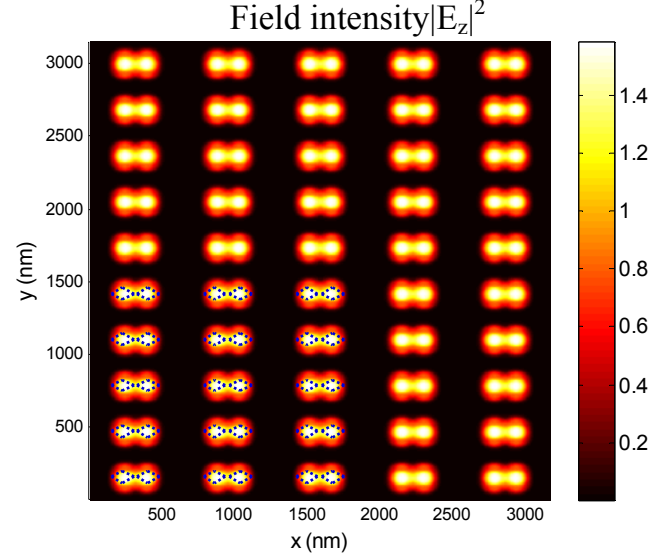

Figure 11: Representative FDTD near-field intensity maps. These maps were calculated for the bare antenna sample with 620-nm illuminating light at the parallel polarization (left column) and the perpendicular polarization (right column). The maps show the intensity patterns $10 \mathrm{~nm}$ above the sample, averaged in a 150-nm radius circle, and plotted for each field component. The corresponding intensity scales (arbitrary units) are also shown. The $\mathrm{x}$ and $\mathrm{z}$ components dominate in the parallel polarization, but the y component dominates in the perpendicular polarization. 


\section{COMPARING FDTD SIMULATIONS TO NSOM: RESULTS AND DISCUSSION}

We can now compare the total, FDTD-calculated intensity distributions $\left|\mathrm{E}_{\text {Total }}\right|^{2}$ to the experimentally measured NSOM results. For each sample (bare antennas, superlens, and reference), we show images of the NSOM scans in the parallel and perpendicular incident light polarizations. The FDTD simulation results for the same polarizations are shown below the corresponding experimental scans. Schematic insets on the NSOM scans show the incident-light polarization and the orientation of the antennas (not to scale). Although all the simulations results have been averaged over an area roughly the size of the NSOM tip aperture, it is still obvious that the simulation results are much clearer than the NSOM measurements. We attribute this loss in resolution in the NSOM scans to a possible larger tip aperture due to inevitable damage during scanning, to sample/tip interactions that perturb the near-field, and/or to imperfections in fabrication. It should be noted that in most of the NSOM measurements, the sample image is rotated roughly 15 degrees from vertical. This slight orientation offset is due to the fact that sample alignment in the NSOM is done manually, and it is therefore quite difficult to align the sample precisely.

\section{Bare Antenna Sample}

We begin with our results for the bare antenna structure in Figure 12. Both the simulations and the NSOM results were performed with illumination at a wavelength of $620 \mathrm{~nm}$, which corresponds to the transmission dip (resonance) observed in our far-field measurements.
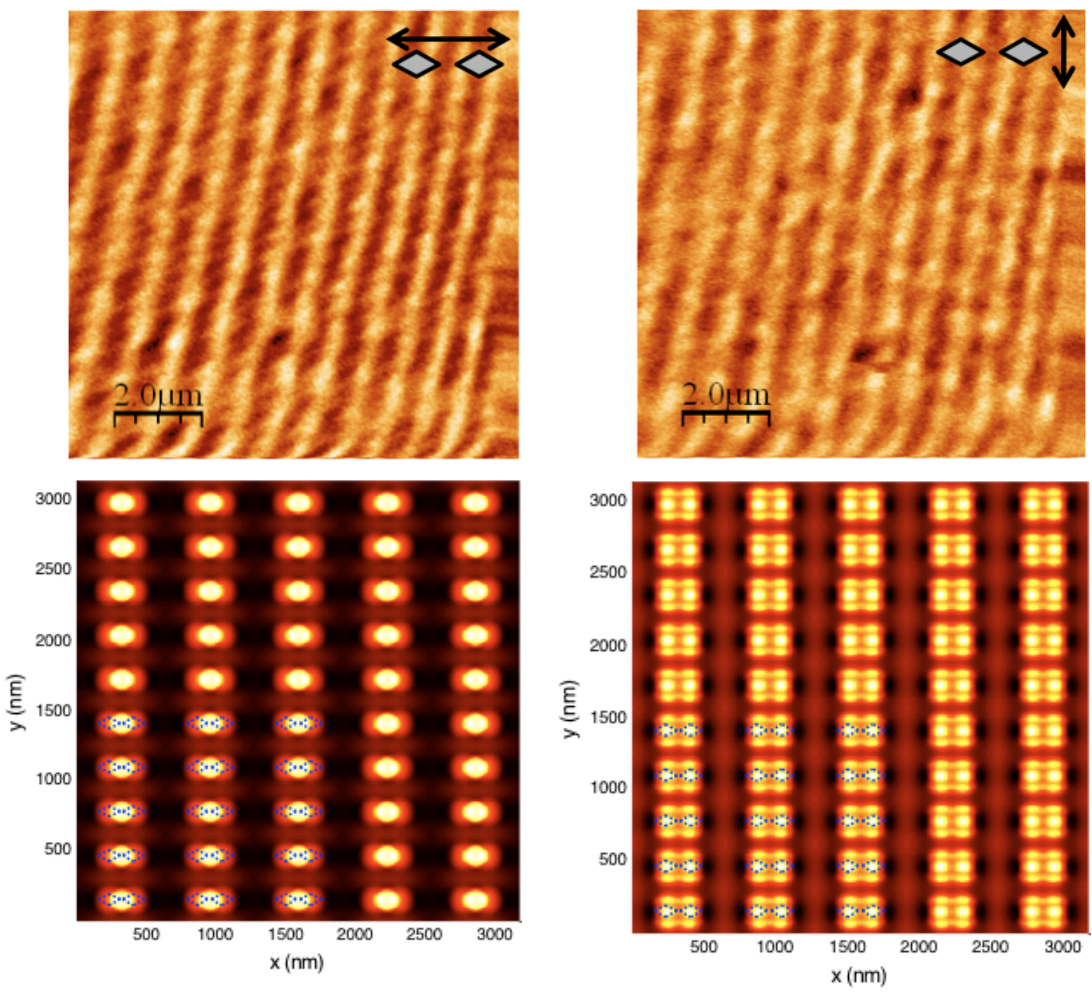

Figure 12: NSOM scans and corresponding total near-field intensity simulations for the bare antenna sample in both polarizations and at an incident wavelength of $620 \mathrm{~nm}$.

Note that, as before, we see stripes in the NSOM images due to the inability to resolve the rows of antenna pairs, although we can resolve the columns of the array. We see very little difference between the NSOM scans for the two polarizations on the bare antenna sample (top row of Figure 12). The only differences are slightly broader high-intensity stripes in the perpendicular polarization than in the parallel polarization. Comparing the FDTD results (bottom row of Figure 12), we see that the parallel-polarized light is strongly confined to the antenna gap, which is expected from the previous far-field measurements, and the intensity does not extend as far in the y direction as in the $\mathrm{x}$ direction. In the 
case of perpendicularly polarized light, the intensity is not localized to the antenna gap, and the intensity profile is broader in both the $\mathrm{x}$ and $\mathrm{y}$ directions. Both of these results agree reasonably well with our NSOM results.

\section{Superlens Sample}

In Figure 13 we show the NSOM scans and simulation results for the superlens sample measured and simulated with 680-nm incident light. In this case, the FDTD simulations correspond very well with the NSOM results. We clearly see that the parallel-polarized light exhibits double periodicity (two intensity peaks per antenna pair), while the perpendicularly polarized light exhibits single periodicity. The double periodicity of the parallel-polarized light can be attributed almost entirely to the $\left|E_{z}\right|^{2}$ component of the field (data not shown). This is indicative of diffraction coming from the antenna structures, rather than the antenna hot-spot transfer that we were seeking. However, the intensity profile still shows subwavelength confinement on the far side of the superlens.
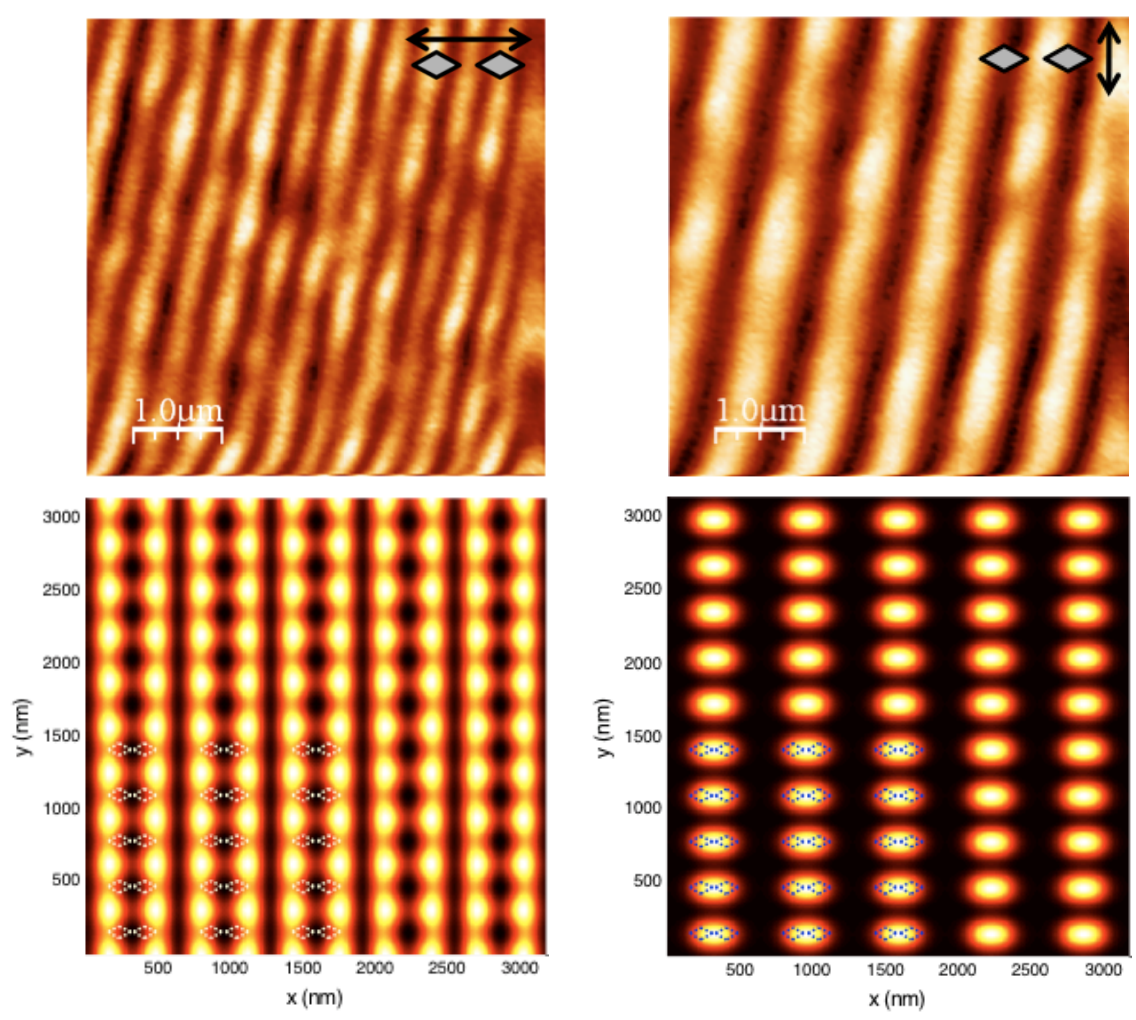

Figure 13: NSOM scans (upper panels) and corresponding total near-field intensity simulations (lower panels) for the superlens sample in both polarizations and at an incident wavelength of $680 \mathrm{~nm}$.

To further validate this hypothesis, scans were taken at Purdue with unpolarized, 633-nm light at the edge of the antenna array, as seen in Figure 14. The left image of Figure 14 shows the AFM height profile that was measured concurrently with the NSOM scan. While the antennas cover only about $40 \%$ of the image, the intensity pattern extends throughout the whole NSOM image. This further indicates that a propagating diffraction mode dominates the near-field intensity profile on the far side of the superlens. 

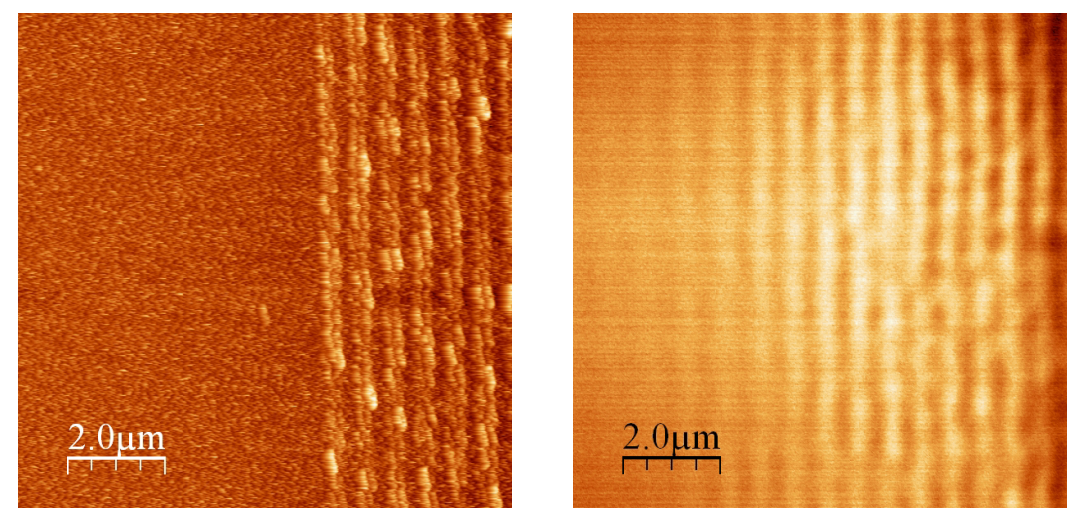

Figure 14: NSOM scans with unpolarized light at the edge of an antenna array. The image on the left shows the topography (height profile) of the sample. The antennas cover roughly $40 \%$ of the image. The image on the right shows the corresponding NSOM intensity profile of the same region. The intensity pattern continues beyond the array, indicating a propagating, diffraction-based mode.

\section{Reference Sample}

Figure 15 shows the experimental NSOM and corresponding simulation images for the reference sample, which has only a dielectric spacer above the nanoantenna arrays. In this case, both the simulations and the NSOM results were found using 660-nm incident light. The FDTD simulations for the reference sample correspond reasonably well with the NSOM results. The simulation for parallel-polarized light shows a very wide and ill-defined profile in the high-intensity stripes, and the NSOM results show similar results with relatively wide, poorly defined stripes. For the case of perpendicularly polarized incident light, the NSOM results clearly show high intensities for the areas adjacent to the individual antenna pairs. These results also correspond quite well with the FDTD simulation, where strong intensities are predicted for the areas adjacent to the antenna pairs. 

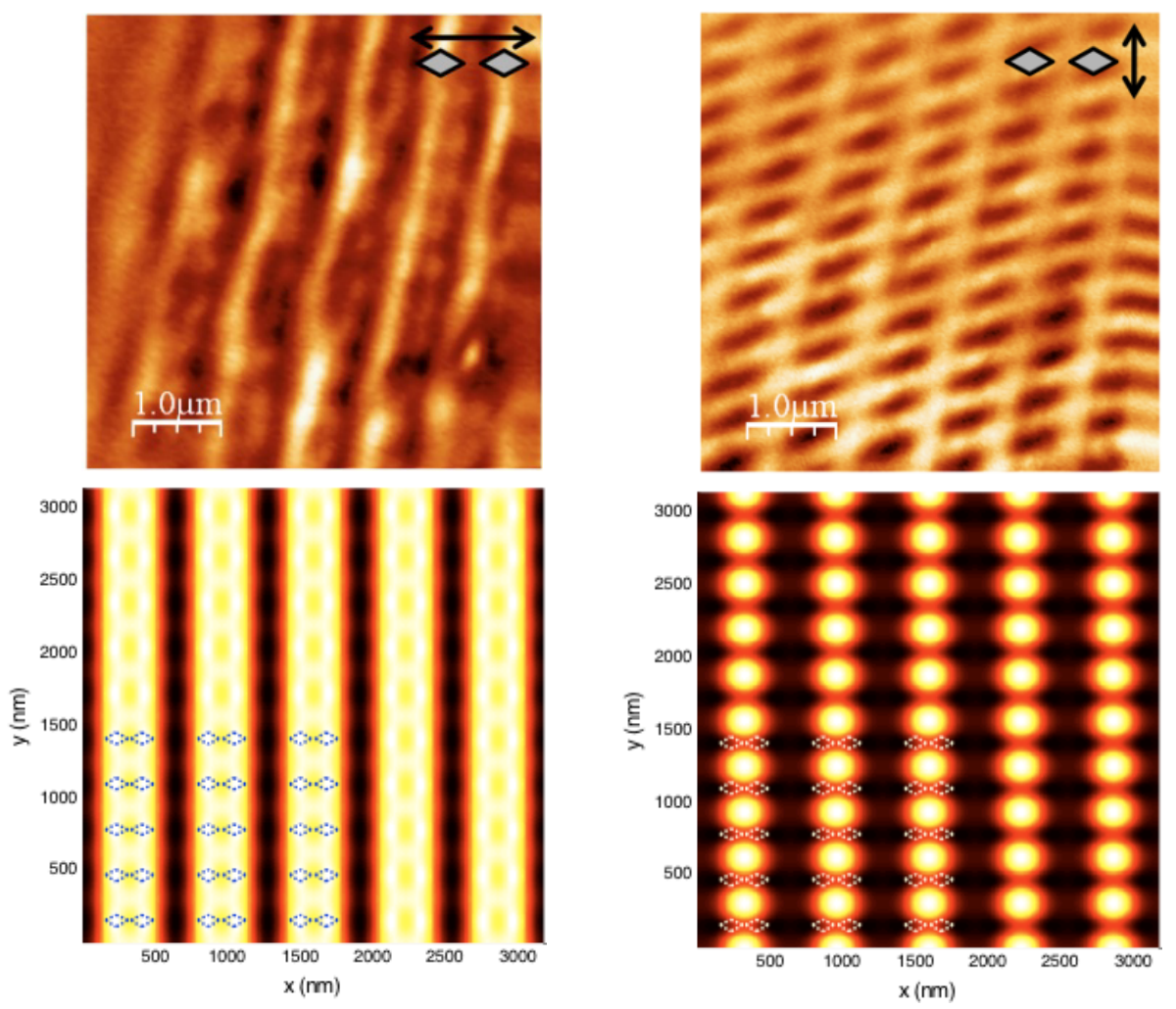

Figure 15: NSOM scans (upper panels) and corresponding total near-field intensity simulations (lower panels) for the reference sample in both polarizations and at an incident wavelength of $660 \mathrm{~nm}$.

\section{CONCLUSIONS AND FINAL REMARKS FOR LAMELLAR SUPERLENS DESIGNS}

We have studied lamellar superlens designs using experimental measurements and advanced numerical simulations. For this work, three different sample types were prepared: bare antennas, antennas with a silver superlens, and antennas with a reference dielectric layer. Each sample was designed, fabricated, and characterized to ascertain how the enhanced-field hot spot created by the antennas was translated to the top side of the sample. In-depth FDTD simulations were performed to compare with experimental measurements, and after analyzing the results, we conclude that the superlens is not strongly transferring the antenna hot spot to the top side of the sample. However, the superlens does provide the unintended consequence of transferring a diffraction grating to the far side of the superlens, and this still provides subwavelength confinement of energy that could be used in enhanced sensing applications or other devices.

In fact, this phenomenon provides a method of tailoring the localized, subwavelength near-field enhancement of a superlens-translated intensity distribution. By adjusting the sizes, shapes and periodicities of the nanoantenna array, we can apply a genetic algorithm to optimize the near-field intensity distribution on the far side of a superlens. In doing so, we can match the final near-field intensity pattern to a specific application such as sensing or nonlinear-optical studies. This will be our next step in our research on nanoantenna-superlens combinations.

\section{ACKNOLEDGEMENTS}

This work was sponsored by the 2009 SAOT Young Researcher Award, Intelligence Community Postdoctoral Research Fellowship Grant \#104796, the Cluster of Excellence Engineering of Advanced Materials (EAM), the Danish Research Council for Technology and Production Sciences (Grant No. 274-07-0057), and ARO-MURI Award W911NF-09-10539. 


\section{REFERENCES}

[1] Pendry, J. B., "Negative refraction makes a perfect lens," Phys. Rev. Lett. 85, 3866 (2000).

[2] Veselago, V. G., "The electrodynamics of substances with simultaneously negative values of $\varepsilon$ and $\mu$," Sov. Phys. Usp. 10, 509 (1968).

[3] Fang, N., Lee, H., Sun, C. and Zhang, X. "Sub-diffraction-limited optical imaging with a silver superlens," Science 308, 534 (2005).

[4] Melville, D. O. S. and Blaikie, R. J., "Super-resolution imaging through a planar silver layer," Optics Express 13, 2127 (2005).

[5] Jeppesen, C., Nielsen, R. B., Boltasseva, A., Xiao, S., Mortensen, N. A. and Kristensen, A., "Thin film superlens towards lab-on-a-chip integration," Optics Express 17, 22543 (2009).

[6] Cai, W., Genov, D. A. and Shalaev, V. M., "Superlens based on metal-dielectric composites," Phys. Rev. B 72 , 193101 (2005).

[7] Kildishev, A. V., Cai, W., Chettiar, U. K., Yuan, H.-K., Sarychev, A. K., Drachev, V. P. and Shalaev, V. M., "Negative refractive index in optics of metal-dielectric composites," J. Opt. Soc. Am. B 23, 423 (2006).

[8] Shi, L., Gao, L., He, S. and Li, B., "Superlens from metal-dielectric composites of nonspherical particles," Phys. Rev. B 76, 045116 (2007).

[9] Shi, L., Gao, L. and He, S., "Tunable negative refraction and subwavelength imaging in the metal-dielectric composites of nonspherical particles,” Proc. Int. Symp. Biophot. Nanophot. Metamat., 463-466 (2006).

[10] Jacob, Z., Alekseyev, L. V. and Narimanov, E., "Optical hyperlens: far-field imaging beyond the diffraction limit," Optics Express 14(18), 8247 (2006).

[11] Belov, P. A. and Hao, Y., "Subwavelength imaging at optical frequencies using a transmission device formed by a periodic layered metal-dielectric structure operating in the canalization regime," Phys. Rev. B 73, 113110 (2006).

[12] Chaturvedi, P. and Fang, N. X., "Molecular scale imaging with a mutlilayer superlens," Mater. Res. Soc. Symp. Proc. 919, 0919-J04-07 (2006).

[13] Wood, B., Pendry, J. B. and Tsai, D. P., "Directed subwavelength imaging using a layered metal-dielectric system," Phys. Rev. B 74, 115116 (2006).

[14] Shamonina, E., Kalinin, V. A., Ringhofer, K. H. and Solymar, L., "Imaging, compression and Poynting vector streamlines for negative permittivity materials," Electron. Lett. 37, 1243 (2001).

[15] Ramakrishna, S. A., Pendry, J. B., Wiltshire, M. C. K. and Stewart, W. J., "Imaging the near field," J. Mod. Opt. 50,1419 (2003).

[16] Aravind, P. K., Nitzan, A. and Metiu, H., "The interaction between electromagnetic resonances and its role in spectroscopic studies of molecules adsorbed on colloidal particles or metal spheres," Surf. Sci. 110, 189 (1981).

[17] Hao, E. and Schatz, G. C., "Electromagnetic fields around silver nanoparticles and dimers," J. Chem. Phys. 120, 357 (2004).

[18] Bakker, R. M., Boltasseva, A., Liu, Z., Pedersen, R. H., Gresillon, S., Kildishev, A. V., Drachev, V. P. and Shalaev, V. M., "Near-field excitation of nanoantenna resonance," Optics Express 15, 13682 (2007).

[19] Shalaev, V. M. and Stockman, M. I., "Optical properties of fractal clusters (susceptibility, surface enhanced Raman scattering by impurities)," Sov. Phys. JETP 65(2), 287 (1987).

[20] Markel, V. A., Muratov, L. S., Stockman, M. I. and George, T. F., "Theory and numerical simulation of optical properties of fractal clusters," Phys. Rev. B 43, 8183 (1991).

[21] Shalaev, V. M., "Electromagnetic properties of small-particle composites," Phys. Rep. 272, 61 (1996).

[22] Podolskiy, V.A., Sarychev, A. K., Narimanov, E. E. and Shalaev, V. M., "Resonant light interaction with plasmonic nanowire systems," J. Opt. A: Pure Appl. Opt. 7, S32 (2005).

[23] Chettiar, U. K., Kildishev, A. V., Klar, T. A. and Shalaev, V. M., "Negative index metamaterial combining magnetic resonators with metal films," Optics Express 14, 7872 (2008).

[24] Liu, Z., Thoreson, M. D., Kildishev, A. V. and Shalaev, V. M., "Translation of nanoantenna hot spots by a metaldielectric composite superlens," Appl. Phys. Lett. 95, 033114 (2009).

[25] Muhlschlegel, P., Eisler, H. J., Martin, O. J. F., Hecht, B. and Pohl, D. W., "Resonant optical antennas," Science 308, 1607 (2005).

[26] Rechberger, W., Hohenau, A., Leitner, A., Krenn, J. R., Lamprecht, B. and Aussenegg, F. R., "Optical properties of two interacting gold nanoparticles," Opt. Comm. 220, 137 (2003).

[27] Farahani, J. N., Pohl, D. W., Eisler, H.-J. and Hecht, B., "Single quantum dot coupled to a scanning optical antenna: a tunable superemitter," Phys. Rev. Lett. 95, 017402 (2005). 
[28] Liu, Z., Boltasseva, A., Pedersen, R. H., Bakker, R., Kildishev, A. V., Drachev, V. P. and Shalaev, V. M., "Plasmonic nanoantenna arrays for the visible," Metamaterials 2, 45 (2008).

[29] Kneipp, K., Moskovits, M. and Kneipp, H., [Surface-Enhanced Raman Scattering], Springer, Berlin (2006).

[30] Bakker, R. M., Drachev, V. P., Liu, Z., Yuan, H.-K., Pedersen, R. H., Boltasseva, A., Chen, J., Irudayaraj, J., Kildishev, A. V. and Shalaev, V. M., "Nanoantenna array-induced fluorescence enhancement and reduced lifetimes," New J. Phys. 10, 125022 (2008).

[31] Holt, R. and Cotton, T., "Surface-enhanced resonance Raman and electrochemical investigation of glucose-oxidase catalysis at a silver electrode," J. Am. Chem. Soc. 111(8), 2815-2821 (1989).

[32] Yang, M., Chung, M., and Thompson, M., "Acoustic network analysis as a novel technique for studying protein adsorption and denaturation at surfaces," Anal. Chem. 65(24), 3713-3716 (1993).

[33] Anger, P., Bharadwaj, P. and Novotny, L., "Enhancement and quenching of single-molecule fluorescence," Phys. Rev. Lett. 96(11), 113002 (2006).

[34] Nielsen, R. B., Thoreson, M. D., Chen, W., Kristensen, A., Hvam, J. M., Boltasseva, A. and Shalaev, V.M., "Toward superlensing with metal-dielectric composites and multilayers," Appl. Phys. B 100, 93-100 (2010), Also: Nielson, R. B., Kristensen, A., Hvam, J. M. and Boltasseva, A., "Metal-dielectric composites with tunable optical properties," Proc. SPIE 7711, 77111Q (2010).

[35] Thoreson, M. D., Fang, J., Kildishev, A. V., Prokopeva, L., Nyga, P., Chettiar, U. K., Shalaev, V. M. and Drachev, V. P., "Fabrication and realistic modeling of three-dimensional metal-dielectric composites," J. Nanophoton. 5, 051513 (2011).

[36] IOFFE Physico-Technical Institute, Electronic Archive "New Semiconductor Materials. Characteristics and Properties," www.ioffe.ru/SVA/NSM/nk/GermaniumCompounds/Gif/ge.gif

[37] Su, K.-H., Wei, Q.-H., Zhang, X., Mock, J. J., Smith, D. R. and Schultz, S., "Interparticle coupling effects on plasmon resonances of nanogold particles," Nano Lett. 3(8), 1087-1090 (2003).

[38] Ebbesen, T. W., Genet, C. and Bozhevolnyi, S. I., "Surface-plasmon circuitry," Physics Today 61(5), 44-50 (2008).

[39] Parriaux, O., Sychugov, V. A. and Tishchenko, A. V., "Optimization and control of grating coupling to or from a silicon-based optical waveguide," Pure Appl. Opt. 5(4), 453-469 (1996).

[40] Prokopeva, L. J., Borneman, J. and Kildishev, A. V., "Optical Dispersion Models for Time-Domain Modeling of Metal-Dielectric Nanostructures,” IEEE Trans. Magn. 47(5), 1150-1153 (2011). 